\title{
Reflection Characteristics of Inhomogeneous Plasma backed by Radar Absorbing Material
}

\author{
Simy Antony and Hema Singh \\ Centre for Electromagnetics (CEM) \\ CSIR-National Aerospace Laboratories \\ Bangalore 560017 \\ Email: hemasingh@nal.res.in
}

\begin{abstract}
The radar cross section reduction can be achieved by controlling the reflections from the surface of the structure. Plasma envelope is one of the ways to control the reflections and scattering from the surface. The problem of EM propagation within bounded plasma can be approximated as multi-layered dielectric problem. The overall reflection from an inhomogeneous plasma on a RAM covered conducting surface is discussed in this paper. The assumed structure is multi-layered, consisting of plastic, plasma, radar absorbing material and conductor. The propagation characteristic of the electromagnetic wave is analyzed using impedance transformation method. The dependence of reflection behavior of the multilayered structure on the plasma parameters is studied. It is shown that the absorption and its bandwidth are improved due to plasma inhomogeneity.

Index Terms - EM propagation, impedance transformation, inhomogeneous plasma, radar absorbing material, reflection.
\end{abstract}

\section{INTRODUCTION}

The metallic targets are easily detectable due to its highly reflecting nature. The reflections can be reduced by the use of low observable techniques. Plasma is one of the choices for controlling the reflections from the surface. Vidmar [1] showed that the cold plasma can absorb electromagnetic (EM) wave. The plasma-based control over the reflection from the structure can be further improved by the inclusion of RAM layer and introducing inhomogeneity in the plasma. In case of open plasma, problems such as plasma trail, visible glow etc. limits its application in stealth techniques. In order to avoid such problems, a plastic envelope is used to cover the plasma, making it bounded plasma. An impedance transformation based on multiple dielectric layered media is employed to study the reflections from bounded homogeneous/ inhomogeneous plasma covering RAM structure [2], [3]. It is noted that inhomogeneous plasma has better performance as compared to homogeneous plasma. This is because of the gradual impedance transition due to the inhomogeneous nature of the plasma (density profile) at the air plasma interface.

In this paper, a four layered structure consisting of plastic, inhomogeneous plasma, RAM and conductor is considered. The overall reflection from the structure includes reflections at the interfaces (air-plasma, plastic-plasma, plasma-RAM, RAM-conductor) and the reflections within the plasma. EM wave propagation characteristics of the structure are studied using impedance transformation method [4]. Inhomogeneous plasma layer is divided into 12 sub-layers with same thickness and plasma collision frequency, but with distinct electron density. Overall electron density in the plasma layer follows specific density profile (linear, parabolic and exponential). The RAM material is assumed to be lossy magnetic in nature.

\section{EM PROPAGATION IN A FOUR-LAYERED PLASMA-RAM STRUCTURE}

Figure 1 shows the schematic of a four-layered plasmaRAM structure. The EM wave, propagating in $-z$ direction, is assumed to normally incident on the plasma layer. The reflections of EM wave at the interfaces (air-plasma, plasmaconductor) are determined in terms of layer parameters, such as thickness, permittivity, and permeability of each layer. The impedance is transformed as EM wave propagates from air to plasma towards the conductor.

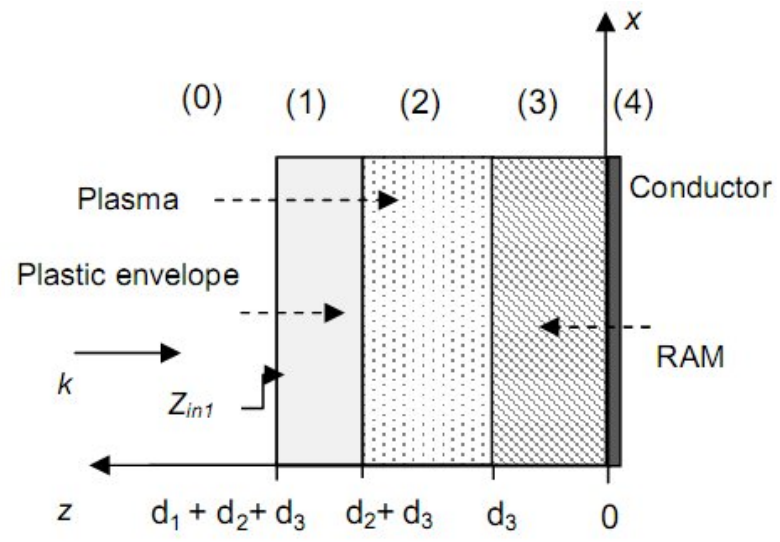

Fig. 1. Four layered plasma-RAM structure

The layer parameters, which controls the propagation characteristics of the EM wave, $Z_{0}, Z_{1}, Z_{2}, Z_{3}, k_{0}, k_{1}, k_{2}, k_{3}, \mu_{0}$, $\mu_{1}, \mu_{2}, \mu_{3}, \varepsilon_{0}, \varepsilon_{1}, \varepsilon_{2}, \varepsilon_{3}$ are the impedance, wave number, permeability and permittivity in $0^{\text {th }}, 1^{\text {st }}, 2^{\text {nd }}$ and $3^{\text {rd }}$ layer respectively. $d_{1}, d_{2}$ and $d_{3}$ are the thickness of $1^{\text {st }}, 2^{\text {nd }}$ and $3^{\text {rd }}$ layer respectively. The incident wave gets reflected at each interface. In plasma layer, the propagating wave will be reflected back and forth at the boundaries and be partially absorbed within the plasma. Cavity resonance effect and plasma collision frequency are the main absorption 
mechanism in the plasma. The dielectric constant of the plasma depends upon the plasma frequency $\left(\omega_{\mathrm{p}}\right)$, plasma collision frequency $\left(V_{\text {en }}\right)$, and can be expressed as [5]

$$
\varepsilon_{2}=\left(1-\frac{\omega_{p}^{2}}{\omega^{2}+V_{e n}^{2}}\right)-j \times \frac{V_{e n}}{\omega} \frac{\omega_{p}^{2}}{\omega^{2}+V_{e n}^{2}} .
$$

The plasma frequency can be determined from the plasma electron density $\left(N_{\mathrm{e}}\right)$,

$$
\omega_{p}=\sqrt{\frac{N_{e} e^{2}}{m_{e} \varepsilon_{o}}} .
$$

For inhomogeneous plasma the sub-layers in the plasma layer have different dielectric constant. This makes the inhomogeneous plasma a multilayered dielectric medium and can be analyzed using impedance transformation method. In each density profile, the electron density increases from the air-plasma interface. In other words, the electron density is minimum at the air-plasma interface and increases gradually to its maximum at the plasma-conductor interface. This makes gradual impedance transformation for the propagating wave travelling. The reflections from the structure can be determined from the impedance of first layer, $Z_{\text {in } 1}$, i.e.

$$
\Gamma=\frac{Z_{i n 1}-Z_{o}}{Z_{i n 1}+Z_{o}} .
$$

where $\Gamma$ is the reflection coefficient, $Z_{\mathrm{o}}$ is the free space impedance. The total reflected power can be calculated as

$$
R=|\Gamma|^{2}
$$

The impedance at $n^{\text {th }}$ interface is expressed as

$$
Z_{i n(n)}=\frac{Z_{i n(n+1)} \cosh j k_{n} d_{n}+Z_{n} \sinh j k_{n} d_{n}}{Z_{i n(n+1)} \sinh j k_{n} d_{n}+Z_{n} \cosh j k_{n} d_{n}} \times Z_{n} .
$$

For an inhomogeneous plasma, electron density can be determined from specific density profile. The electron density profile in the $n^{\text {th }}$ plasma sub-layer with linear, parabolic and exponential density profiles are expressed as

Linear: $N_{e}(n)=\frac{N_{e}(t-(n-1))}{t}$

Parabolic:

$$
\begin{aligned}
& N_{e}(n)=N_{e} \exp \frac{2}{3}\left(\frac{2(t-(n-1))}{t}-1\right) ; Z<\frac{d}{2} \\
& N_{e}(n)=N_{e} \exp \frac{2}{3}\left(1-\frac{2(t-(n-1))}{t}\right) ; \frac{d}{2}<z<d
\end{aligned}
$$

Exponential:

$$
N_{e}(n)=N_{e} \exp \frac{2}{3}\left(\frac{(t-(n-1))}{t}-1\right)
$$

where $t$ is the number of sub-layers in the plasma, and $d$ is the plasma thickness.

\section{RESULTS AND DISCUSSION}

In this section, reflection characteristics of a four-layered structure consisting of plastic, inhomogeneous plasma, RAM and conductor is discussed. The main absorption of EM wave occurs in plasma and RAM layer. For an efficient absorption, matching between RAM and plasma parameters is required. The permittivity and permeability of the lossy magnetic RAM layer is given by

$$
\begin{gathered}
\varepsilon_{3}=15 \\
\mu_{3}=\frac{306.25}{f_{g}^{2}+12.25}-i \times \frac{87.5 \times f_{g}}{f_{g}^{2}+12.25}
\end{gathered}
$$

where $f_{\mathrm{g}}=f \times 10^{-9}$. The reflection characteristic of the inhomogeneous plasma with linear electron density profile is shown in Fig. 2.

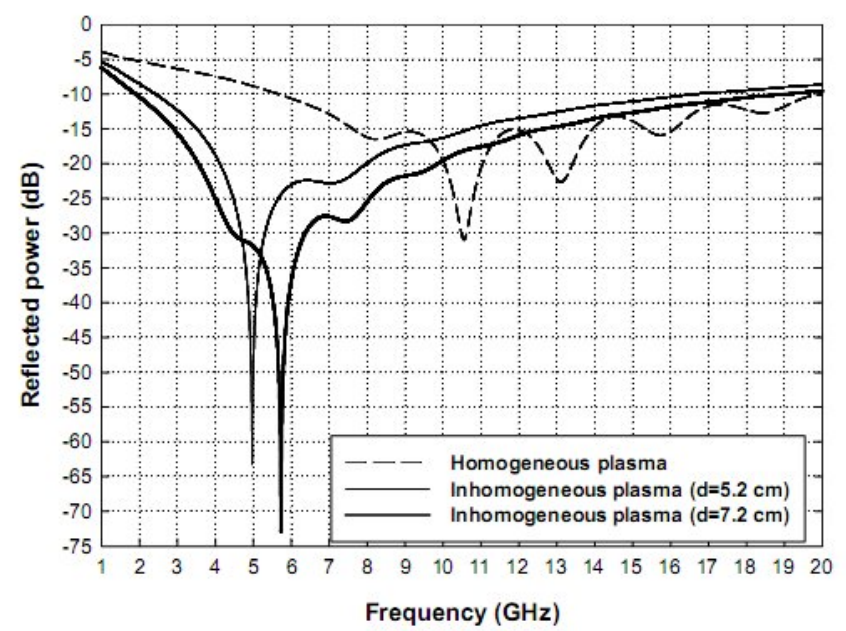

Fig. 2. Comparison of reflection characteristics of four-layered structure having homogeneous and inhomogeneous plasma with linear electron density profile, $V_{\mathrm{en}}=30 \mathrm{GHz}, N_{\mathrm{e}}=5 \times 10^{17} \mathrm{~m}^{-3}$.

The thickness of Layer 1 (plastic) and Layer 3 (RAM) is taken as $1 \mathrm{~cm}$. It is to be noted that $N_{\mathrm{e}}$ is the maximum electron density in inhomogeneous plasma. It is apparent from Fig. 2 that the plasma inhomogeneity alters the reflection characteristics of the structure. There is only one absorption peak in case of inhomogeneous plasma. Moreover the inhomogeneity in the plasma shifts the resonant absorption band towards low incident frequency region as compared to homogeneous plasma. At the plastic-plasma interface the electron density is small and hence the wave with low incident frequency can enter into the plasma and get absorbed. The absorption bandwidth increases due to the inhomogeneity in the plasma. It may be observed that for $d_{2}=7.2 \mathrm{~cm}$ the absorption is comparatively more and at higher incident frequency with greater bandwidth.

Figure 3 shows the effect of maximum electron density on the reflected power of four-layered structure having inhomogeneous plasma with linear electron density profile. 
The absorption peak shifts to high incident frequency with greater bandwidth for high electron density.

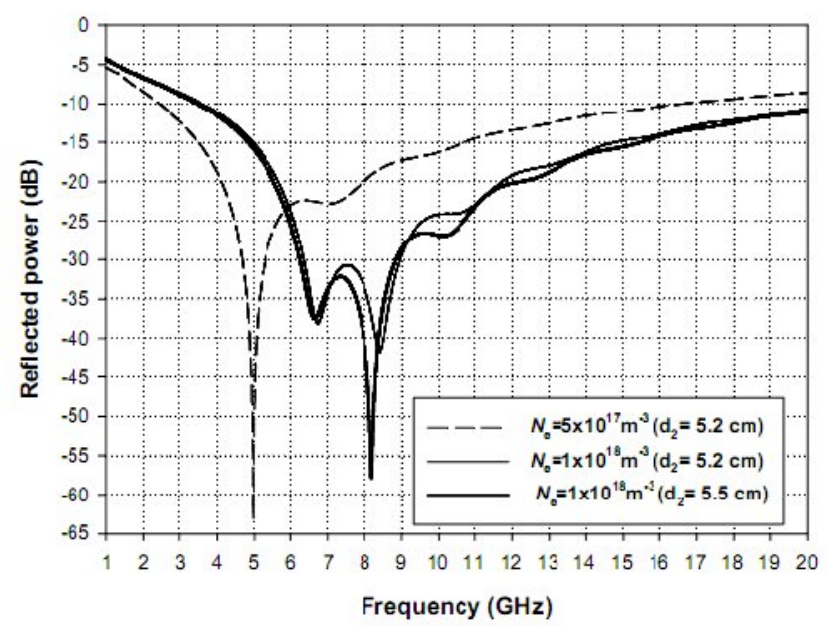

Fig. 3. Effect of maximum plasma electron density on the reflected power of the four-layered structure with inhomogeneous plasma with linear electron density profile, $V_{\mathrm{en}}=30 \mathrm{GHz}$.

In Fig. 4, the effect of plasma collision frequency on the reflected power of four-layered plasma-RAM structure is shown. The inhomogeneous plasma with linear electron density profile is considered. As the collision frequency increases, the absorption peak shifts slightly towards the lower incident frequency region. It can be noted that the optimized plasma thickness for maximum EM wave absorption changes for different plasma electron density and collision frequency. One can infer that the inhomogeneity of the plasma is more effective when the plasma thickness is more.

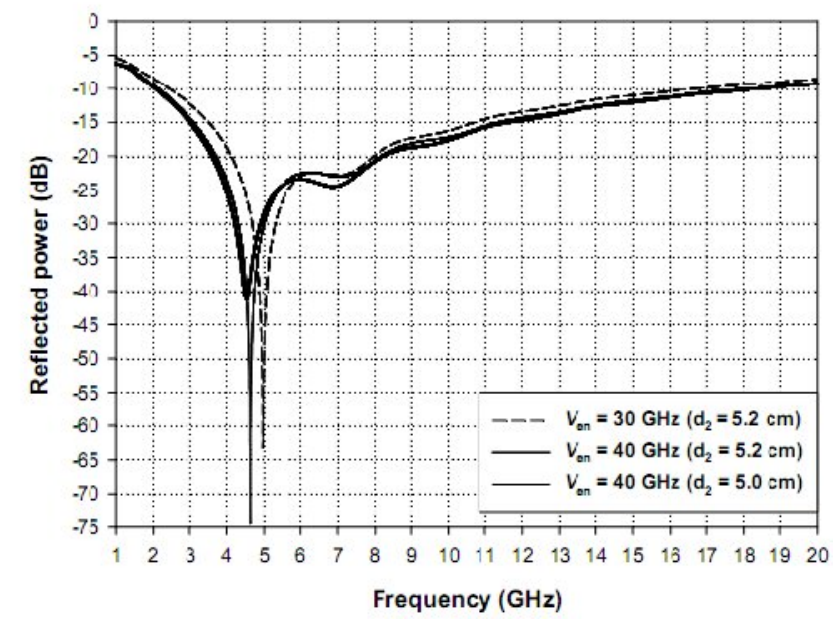

Fig. 4. Effect of plasma collision frequency on the reflected power of the four-layered structure with inhomogeneous plasma with linear electron density profile, $N_{\mathrm{e}}=5 \times 10^{17} \mathrm{~m}^{-3}$.

Figure 5 compares the absorption in four-layered plasmaRAM structure with homogeneous and inhomogeneous plasma. It is noted that due to electron density variation in the plasma region, the resonant frequency shift towards low incident frequency. The shift in resonant frequency towards low frequency is less in parabolic and exponential density profile case compared to linear density profile. This may be due to the fact that for linear profile the electron density at the plastic-plasma interface is less as compared to parabolic and exponential profile.

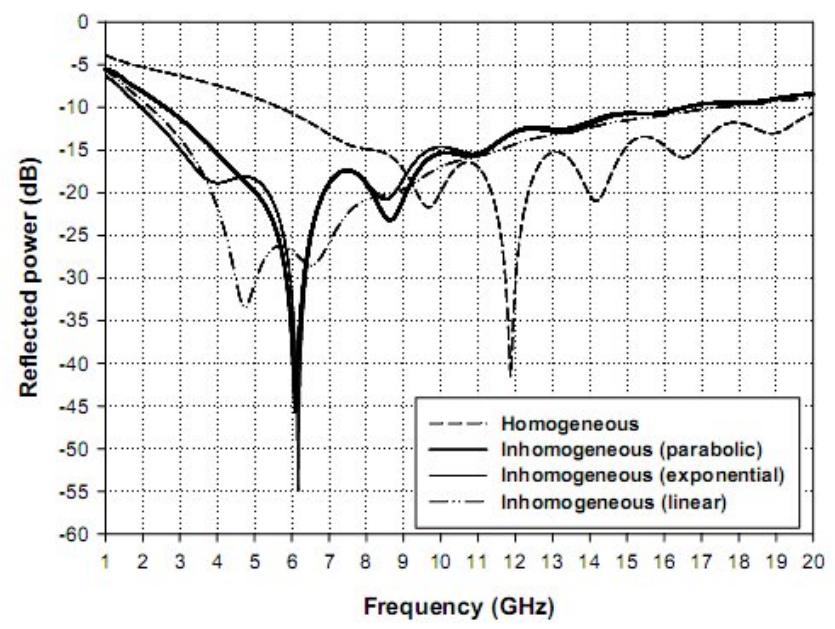

Fig. 5. Comparison of reflection characteristics of four-layered structure having homogeneous and inhomogeneous plasma, $d_{2}=5.9$ $\mathrm{cm}, V_{\mathrm{en}}=30 \mathrm{GHz}, N_{\mathrm{e}}=5 \times 10^{17} \mathrm{~m}^{-3}$.

\section{CONCLUSION}

The reflection behavior of a four-layered plasma-RAM structure is analyzed using impedance transformation method. Absorption in the structure can be controlled by choosing plasma parameters appropriately. The inhomogeneity in plasma widens the absorption band. Here normal incidence is considered. The absorption behavior will change for an obliquely incidence. Further the temperature of plasma can change the reflection characteristics. In inhomogeneous plasma, the incident angle of the propagating EM wave within the plasma layer might vary.

\section{REFERENCES}

[1] R. J. Vidmar, "On the use of atmospheric pressure plasmas as electromagnetic reflectors and absorbers," IEEE Trans. Plasma Sc., vol. 18, no. 4, pp. 733-741, Aug. 1990.

[2] C. X. Yuan, Z. X. Zhou, and H. G. Sun, "Reflection properties of electromagnetic wave in a bounded plasma slab," IEEE Trans. Plasma Sc., vol. 38, no. 12, pp. 3348-3355, Dec. 2010.

[3] C. X. Yuan, Z. X. Zhou, J. W. Zhang, X. L. Xiang, Y. Feng, and H.G. Sun, "Properties of propagation of electromagnetic wave in a multilayer radar absorbing structure with plasma and radar absorbing material," IEEE Trans. Plasma Science, vol. 39, no. 9, pp. 1768-1775, Sep. 2011.

[4] W. H. Hayt, and J.A. Buck, Engineering Electromagnetics. New York: McGraw-Hill Publishing, 608 p., 2011.

[5] A. Fridman, and L. A. Kennedy, Plasma Physics and Engineering. New York: CRC press, 882 p., 2004. 